\title{
Safety and efficacy of 10-fraction hypofractionated radiation therapy for non-small cell lung cancer
}

\author{
Ye Jin Yoo, Su Ssan Kim, Si Yeol Song, Jong Hoon Kim, Seung Do Ahn, Sang-wook Lee, Sang Min Yoon, \\ Young Seok Kim, Jin-hong Park, Jinhong Jung, Eun Kyung Choi \\ Department of Radiation Oncology, Asan Medical Center, University of Ulsan College of Medicine, Seoul, Korea
}

Received: April 7, 2021

Revised: May 28, 2021

Accepted: June 9, 2021

\section{Correspondence:}

Su Ssan Kim

Department of Radiation Oncology, Asan Medical Center, University of Ulsan College of Medicine, 88

Olympic-ro 43-gil, Songpa-gu, Seoul 05505, Korea

Tel: $+82-2-3010-5680$

Fax: +82-2-3010-6950

E-mail:watermountain@hanmail.net ORCID:

https://orcid.org/0000-0002-8473-302X
Purpose: To investigate the safety and efficacy of hypofractionated radiation therapy (HFRT) in patients with non-small cell lung cancer who are unfit for surgery or stereotactic body radiation therapy (SBRT) at our institution.

Materials and Methods: From May 2007 to December 2018, HFRT was used to treat 68 lesions in 64 patients who were unsuitable for SBRT because of central tumor location, large tumor size, or contiguity with the chest wall. The HFRT schedule included a dose of 50-70 Gy delivered in 10 fractions over 2 weeks. The primary outcome was freedom from local progression (FFLP), and the secondary endpoints included overall survival (OS), disease-free survival, and toxicities.

Results: The median follow-up period was 25.5 months (range, 5.3 to 119.9 months). The FFLP rates were $79.8 \%$ and $67.8 \%$ at 1 and 2 years, respectively. The $\mathrm{OS}$ rates were $82.8 \%$ and $64.1 \%$ at 1 and 2 years, respectively. A larger planning target volume was associated with lower FFLP ( $p=0.023)$. Dose escalation was not associated with FFLP $(p=0.964)$. Four patients $(6.3 \%)$ experienced grade $3-5$ pulmonary toxicities. Tumor location, central or peripheral, was not associated with either grade 3 or higher toxicity.

Conclusion: HFRT with 50-70 Gy in 10 fractions demonstrated acceptable toxicity; however, the local control rate can be improved compared with the results of SBRT. More studies are required in patients who are unfit for SBRT to investigate the optimal fractionation scheme.

Keywords: Non-small cell lung carcinomas, Radiation dose fractionation, Radiation dose hypofractionation, Treatment outcome, Toxicity

\section{Introduction}

Stereotactic body radiation therapy (SBRT) has become the treatment of choice for node-negative non-small cell lung cancer (NSCLC) in patients who are medically inoperable or refuse surgery $[1,2]$. SBRT has been reported to result in local tumor control of $>90 \%$ at 3 years in multi-center, prospective trials [3-5]. However, high risks of grade 3-5 toxicities have been reported in cases in which the tumor location was central or adjacent to the chest wall. Central tumors within $2 \mathrm{~cm}$ from the proximal bronchial tree demonstrated $46 \%$ of severe toxicity in 2 years compared with
$17 \%$ in peripheral tumors after SBRT [6]. Similarly, 33\% of grade 3-5 pulmonary toxicities, including bronchial stricture and secondary obstructive pneumonia, were reported in SBRT of central tumors [7]. Additionally, after SBRT for peripheral lung tumors adjacent to the chest wall, approximately $40 \%$ of patients experienced rib fracture in 2 years after the treatment [8]. Most prospective series regarding SBRT include small tumors under $5 \mathrm{~cm}$, and large tumors (>5 cm) often do not satisfy the normal tissue constraints. Some retrospective studies have demonstrated the outcomes of SBRT in large tumors; however, only a minority of such cases were $>5 \mathrm{~cm}$, and a dose of $50 \mathrm{~Gy}$ in 10 fractions was administered when 
normal organ constraints were not met $[9,10]$. Large tumors treated with conventional radiation therapy (RT) regimens were also associated with lower control rates and higher toxicity [11-13].

Therefore, hypofractionated radiation therapy (HFRT) has been suggested for tumors in these cases. Several schemes have been suggested; however, there is no consensus on the fractionation number or size in such cases. Ten-fraction HFRT schedules that range from 50 to 70 Gy are used in our institution. In this analysis, we aimed to investigate the safety and efficacy of 10-fraction HFRT in patients with primary or recurrent NSCLC who are unfit for surgery or SBRT at our institution.

\section{Materials and Methods}

\section{Patients}

We retrospectively reviewed 68 lesions that were treated with 10 fractions of RT with a curative aim in 64 patients at our hospital between May 2007 and December 2018. The study was approved by the Institutional Review Board of the Asan Medical Center (No. 2020-1605), and the requirement for informed consent was waived because of the retrospective nature of the study. The patients were unsuitable for SBRT because of central tumor location, large tumor size, or contiguity with the chest wall. Centrally located tumor was defined as a tumor within $2 \mathrm{~cm}$ from the proximal bronchial tree and tumor immediately adjacent to the mediastinal or pericardial pleura.

The exclusion criteria were as follows: patients with distant metastasis, and patients diagnosed with another cancer within 5 years from the RT. For appropriate staging, physical examination, pathologic confirmation, chest computed tomography (CT), 18-fluoro-deoxyglucose positron emission tomography, and brain magnetic resonance imaging were performed. Before the treatment, pulmonary function tests were performed and lymph node metastases were confirmed histopathologically, whenever possible. The stage was determined according to the American Joint Committee on Cancer 8th edition TNM stage classification.

\section{Treatments and follow-up}

For the planning of treatment, four-dimensional CT was used to measure respiratory tumor motion (slice thickness, $2.5 \mathrm{~mm}$ ). The gross tumor volume (GTV) was delineated on axial CT images using the lung window setting at end of expiration. The internal target volume was contoured in maximum intensity projection images or integrated using each GTV in breathing phases within the gating window. The planning target volume (PTV) margin was determined by adding 5-7 $\mathrm{mm}$ radially and 7-10 $\mathrm{mm}$ longitudinally. Elective nodal irradiation was not performed. The prescribed dose was ad- ministered such that 95\% of PTV received 95\%-100\% of the prescription dose and avoided hot spots in organs-at-risk. Respiratory-gated volumetric-modulated arc therapy or intensity-modulated radiotherapy technique using 6 or 15 MV energy with 5-10 fields was used. The HFRT schedule included a dose of 50-70 Gy in 10 fractions over 2 weeks based on the decision of the radiation oncologist. At each treatment, kV cone-beam CT scans were performed to localize treatment targets and subsequently, two orthogonal fluoroscopic $\mathrm{kV}$ images were obtained to confirm the respiratory motion of the visible mass or carina.

\section{Follow-up, outcomes, and statistical analyses}

Patients were evaluated every week using complete blood count tests and chest X-rays (CXRs). Patients were followed up at 1 month after the treatment, every 3 months during the first 2 years after treatment, and every 6 months until 5 years thereafter using chest $\mathrm{CT}, \mathrm{CXR}$, and laboratory tests.

The primary outcome was freedom from local progression (FFLP), and the secondary endpoints included overall survival (OS), disease-free survival (DFS), and toxicities. The response was assessed using the Response Evaluation Criteria in Solid Tumors (RECIST) criteria and toxicity during and after the treatment was assessed according to the Common Terminology Criteria for Adverse Events version 5.0.

Local progression was defined as failure in the primary tumor site. FFLP is the period of absence of local progression from the date of RT initiation. PFS rates are calculated from the date of RT initiation until recurrence, death, or last follow-up. FFLP, DFS, and OS were calculated using the Kaplan-Meier method. Univariate analysis was performed using log-rank test, and chi-square test was used to identify risk factors for toxicity. Statistical analyses were performed using SPSS version 22.0 (IBM Corp., Armonk, NY, USA).

\section{Results}

\section{Patients and treatments}

Overall, 68 lesions in 64 patients were included in this study, and the characteristics of patients and the disease are listed in Tables 1 and 2, respectively. The median age was 72 years (range, 57 to 89 years). In 68 tumors, 13 (19.1\%) included double primary lung cancer; of them, two patients were treated with HFRT and SBRT for each lesion, one patient underwent surgery for another lesion, one patient received photodynamic therapy, one patient rejected treatment for the other lesion and was only followed up according to his will, and four patients were treated with HFRT for both lesions. Additionally, eight cases were of recurrent NSCLC after surgery or definitive concurrent chemoradiation therapy (CCRT) or SBRT. We in- 
Table 1. Patient characteristics $(n=64)$

\begin{tabular}{lc}
\hline Characteristic & Value \\
\hline Age $(\mathrm{yr})$ & $72(57-89)$ \\
Sex & $59(92.2)$ \\
$\quad$ Male & $5(7.8)$ \\
$\quad$ Female & \\
ECOG performance status & $34(53.1)$ \\
$0-1$ & $30(46.9)$ \\
$2-3$ & \\
Pulmonary function test & $65(20-116)$ \\
FEV1 (\%) & $58(36-111)$ \\
DLCO (\%) & \\
Underlying lung disease & $25(39.1)$ \\
Yes & $39(60.9)$ \\
No
\end{tabular}

Values are presented as median (range) or number (\%).

ECOG, Eastern Cooperative Oncology Group; FEV1, forced expiratory volume in 1 second; DLCO, diffusing capacity of the lungs for carbon monoxide.

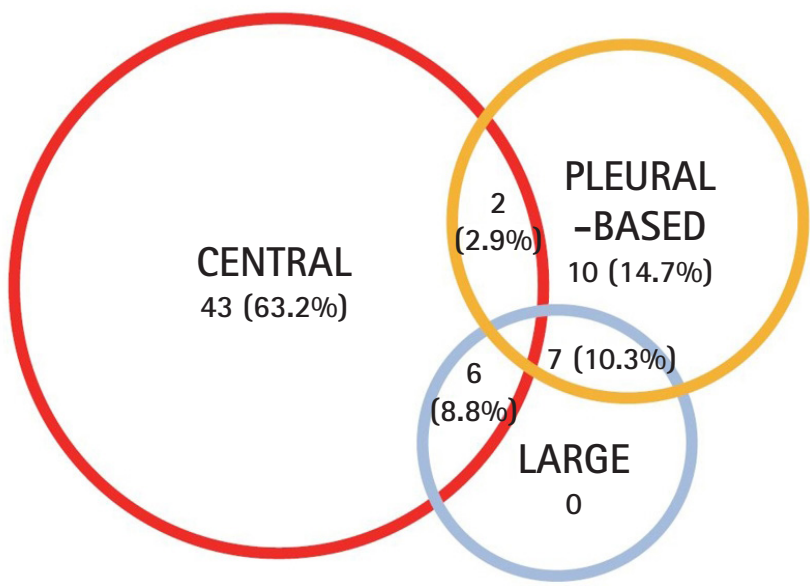

Fig. 1. Reason for hypofractionated radiotherapy.

cluded these patients, and FFLP was calculated based on 68 lesions.

Overall, 51 tumors (75.0\%) were centrally located; of them, 31 cases were ultracentral, which is abutting to the proximal bronchial tree, while the remaining 20 cases were located non-ultra-centrally. Histologically, 41 tumors (64.7\%) were squamous cell carcinoma and 19 tumors (27.9\%) adenocarcinoma. The median size was $3.2 \mathrm{~cm}$ (range, 2.6 to $4.7 \mathrm{~cm}$ ) and 13 tumors (19.1\%) were $>5$ $\mathrm{cm}$ and 55 tumors (80.9\%) were $\leq 5 \mathrm{~cm}$. The median total RT dose was $60 \mathrm{~Gy}$ (range, 50 to $70 \mathrm{~Gy}$ ), and the median biologic equivalent dose (BED) was $96.0 \mathrm{~Gy}_{10}$ (range, 60.0 to $180.0 \mathrm{~Gy}_{10}$ ). Twenty-five tumors (36.8\%) were treated with $50 \mathrm{~Gy}$, one tumor (1.5\%) with 55 Gy, 27 tumors (39.7\%) with $60 \mathrm{~Gy}$, two tumors (2.9\%) for $65 \mathrm{~Gy}$, and 13 tumors (19.1\%) with $70 \mathrm{~Gy}$. The median volumes of GTV and PTV were $18.1 \mathrm{~cm}^{3}$ (range, 0.8 to $161.7 \mathrm{~cm}^{3}$ ) and $60.2 \mathrm{~cm}^{3}$ (range,
Table 2. Disease characteristics and treatment parameters $(n=68)$

\begin{tabular}{|c|c|c|}
\hline Category & & Value \\
\hline \multirow{27}{*}{$\begin{array}{l}\text { Disease } \\
\text { characteristic }\end{array}$} & AJCC 8th stage & \\
\hline & T1N0 & $20(29.4)$ \\
\hline & T2No & $17(25.0)$ \\
\hline & T3NO & $8(11.8)$ \\
\hline & T4N0 & $2(2.9)$ \\
\hline & Double primary lung cancer & $13(19.1)$ \\
\hline & Recurrent NSCLC & $8(11.8)$ \\
\hline & Tumor location & \\
\hline & Central & $51(75.0)$ \\
\hline & Ultracentral & $31(45.6)$ \\
\hline & Non-ultracentral & $20(29.4)$ \\
\hline & Non-central & $17(25.0)$ \\
\hline & Histology & \\
\hline & Squamous cell carcinoma & $44(64.7)$ \\
\hline & Adenocarcinoma & $19(27.9)$ \\
\hline & Others & $2(2.9)$ \\
\hline & Not checkable & $3(4.4)$ \\
\hline & Tumor location & \\
\hline & RUL & $17(25.0)$ \\
\hline & RML & $1(1.5)$ \\
\hline & RLL & $12(17.6)$ \\
\hline & LUL & $19(27.9)$ \\
\hline & LLL & $18(26.5)$ \\
\hline & Regional node (right hilar) & $1(1.5)$ \\
\hline & Tumor size $(\mathrm{cm})$ & $3.2(2.6-4.7)$ \\
\hline & $\leq 5$ & 55 (80.9) \\
\hline & $>5$ & $13(19.1)$ \\
\hline \multirow{8}{*}{$\begin{array}{l}\text { Treatment } \\
\text { parameter }\end{array}$} & Dose (BED) & \\
\hline & 50-60 Gy (75-96 Gy ) & 53 (77.9) \\
\hline & 65-70 Gy (107.25-119 Gy) & $15(22.0)$ \\
\hline & Volume & \\
\hline & GTV $\left(\mathrm{cm}^{3}\right)$ & $18.1(0.8-161.7)$ \\
\hline & PTV $\left(\mathrm{cm}^{3}\right)$ & $60.2(12.5-502.1)$ \\
\hline & Mean lung dose (Gy) & $4.71(1.79-14.74)$ \\
\hline & $\mathrm{V}_{20}(\%)$ & $6.7(1.5-29.2)$ \\
\hline
\end{tabular}

Values are presented as number (\%) or median (range).

AJCC, American Joint Committee on Cancer; NSCLC, non-small cell lung cancer; RUL, right upper lobe; $R M L$, right middle lobe; $R L L$, right lower lobe; LUL, left upper lobe; LLL, left lower lobe; BED, biologic equivalent dose; GTV, gross tumor volume; PTV, planning target volume; $\mathrm{V}_{20}$, lung volume dose receiving $\geq 20 \mathrm{~Gy}$.

12.5 to $502.1 \mathrm{~cm}^{3}$ ), respectively. The mean lung dose was $4.71 \mathrm{~Gy}$ (range, 1.79 to $14.74 \mathrm{~Gy}$ ), and the volume of lung that received at least $20 \mathrm{~Gy}\left(\mathrm{~V}_{20}\right)$ was $6.7 \%$ (range, $1.5 \%$ to $29.2 \%$ ). The reasons for HFRT are illustrated in Fig. 1. They were as follows: (1) central lesion ( $n=43 ; 63.2 \%$ ); (2) pleural-based lesion ( $n=10 ; 14.7 \%$ ); (3) central and pleural-based lesion ( $n=2 ; 2.9 \%)$; (4) central and large 
mass > $5 \mathrm{~cm}(\mathrm{n}=6 ; 8.8 \%)$; and (5) large mass $>5 \mathrm{~cm}$ and pleural-based lesion ( $n=7 ; 10.3 \%$ ). No patient was treated with adjuvant chemotherapy after HFRT.

\section{FFLP, DFS, OS, and failure pattern}

The median follow-up duration was 25.5 months (range, 5.3 to 119.9 months). The FFLP rates were 79.8\% and $67.8 \%$ at 1 and 2 years, respectively (Fig. 2A). The DFS rates were $54.7 \%$ and $32.8 \%$ and the OS rates were $82.8 \%$ and $64.1 \%$ at 1 and 2 years, respec- tively (Fig. 2B, 2C). The median OS was 33.7 months (range, 5.3 to 131.4 months). The freedom from distant metastasis rates were 79.4\% and 65.8\% at 1 and 2 years, respectively (Fig. 2D). As shown in Table 3, a PTV volume $>60 \mathrm{~cm}^{3}$ was a significant factor of FFLP in univariate analysis ( $\leq 60$ vs. $>60 \mathrm{~cm}^{3}: 2$-year FFLP, $81.5 \%$ vs. $53.9 \%$, respectively; $p=0.023$ ). There was no statistical difference in the 2-year FFLP rate between the histologic types (squamous cell carcinoma vs. others: $65.5 \%$ vs. $71.8 \%$, respectively; $p=0.273$ ), tumor location (central vs. non-central: $63.8 \%$ vs. $81.4 \%$, respec-

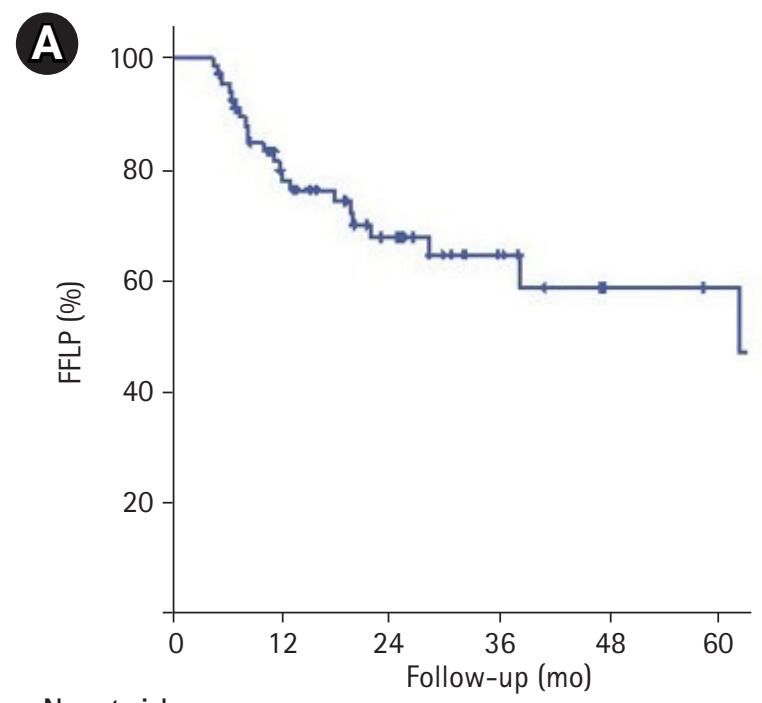

No. at risk

$\begin{array}{llllll}68 & 55 & 49 & 48 & 47 & 47\end{array}$

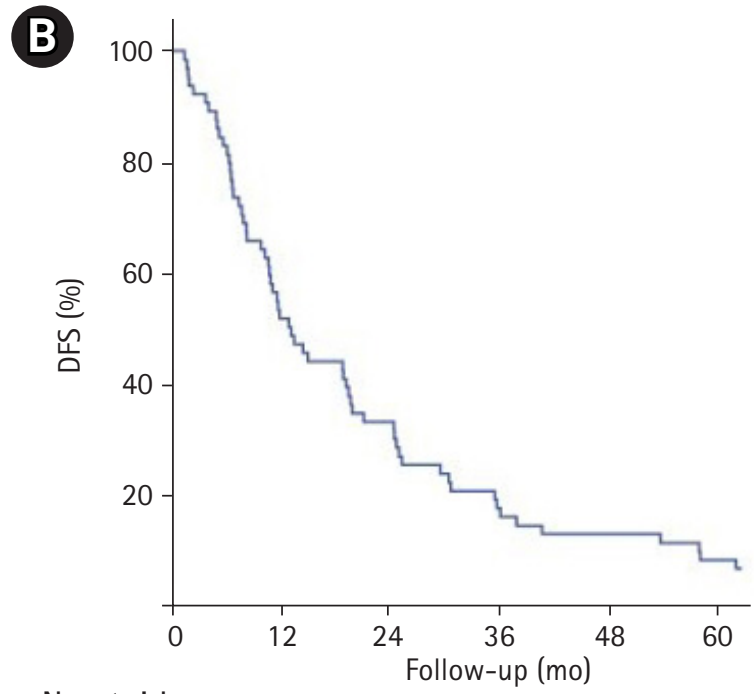

No. at risk

$\begin{array}{llllll}64 & 35 & 21 & 12 & 8 & 5\end{array}$

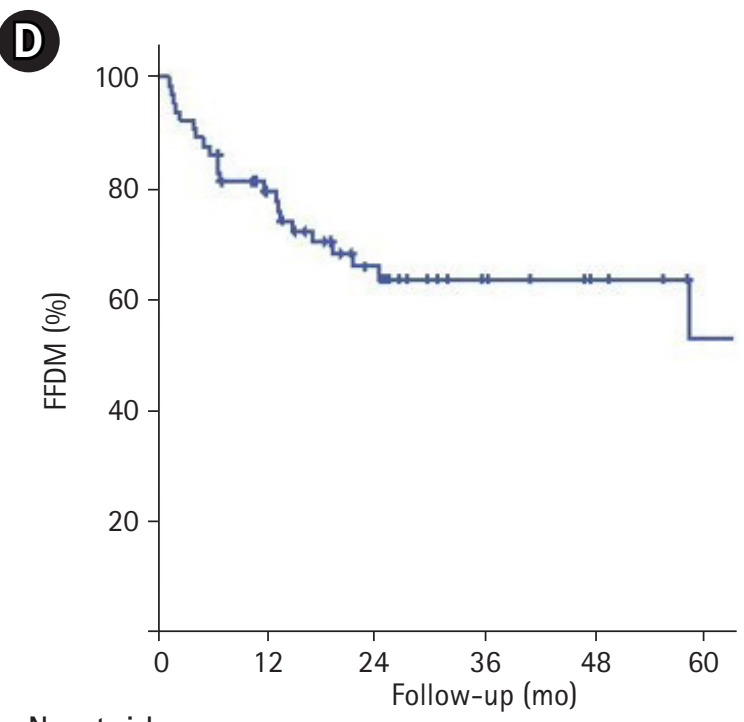

No. at risk

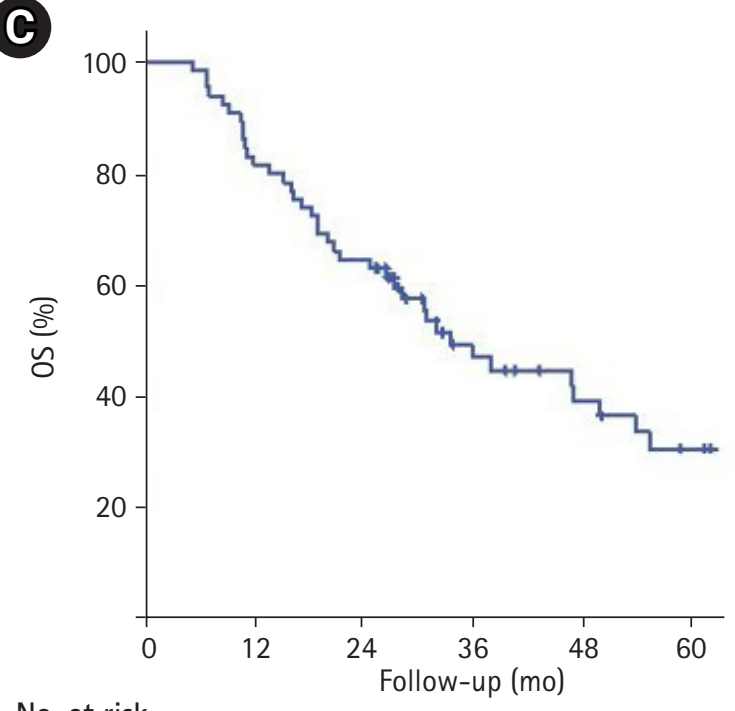

$\begin{array}{llllll}64 & 53 & 41 & 33 & 29 & 26\end{array}$

42

Fig. 2. Survival outcomes of 10-fraction hypofractionated radiotherapy: (A) freedom from local progression (FFLP), (B) disease-free survival (DFS), (C) overall survival (OS), (D) freedom from distant metastasis (FFDM). 
Table 3. Univariate analysis for FFLP in 68 tumors

\begin{tabular}{|c|c|c|c|}
\hline \multirow{2}{*}{ Variable } & \multirow{2}{*}{$\begin{array}{l}\text { Number of } \\
\text { tumors }\end{array}$} & \multicolumn{2}{|c|}{ Univariate analysis } \\
\hline & & 2-yr FFLP (\%) & $p$-value \\
\hline Age (yr) & & & 0.938 \\
\hline$<70$ & 26 & 79.6 & \\
\hline$\geq 70$ & 42 & 80.2 & \\
\hline Sex & & & 0.217 \\
\hline Male & 62 & 64.4 & \\
\hline Female & 6 & 100 & \\
\hline Histologic type & & & 0.273 \\
\hline $\mathrm{SqCC}$ & 44 & 65.5 & \\
\hline Others & 24 & 71.8 & \\
\hline Tumor location & & & 0.288 \\
\hline Central & 51 & 63.8 & \\
\hline Non-central & 17 & 81.4 & \\
\hline Tumor size (cm) & & & 0.473 \\
\hline$\leq 5$ & 55 & 69.9 & \\
\hline$>5$ & 13 & 57.7 & \\
\hline PTV volume $\left(\mathrm{cm}^{3}\right)$ & & & 0.023 \\
\hline$\leq 60$ & 34 & 81.5 & \\
\hline$>60$ & 34 & 53.9 & \\
\hline Dose (Gy) & & & 0.746 \\
\hline $50-60$ & 53 & 66.8 & \\
\hline $65-70$ & 15 & 70.6 & \\
\hline
\end{tabular}

FFLP, freedom from local progression; SqCC, squamous cell carcinoma; PTV, planning target volume.

tively; $p=0.288$ ), and tumor size ( $\leq 5$ vs. $>5 \mathrm{~cm}: 69.9 \%$ vs. $57.7 \%$, respectively; $p=0.473)$. Additionally, there was no difference in FFLP according to dose escalation (50-55 vs. $60-65$ vs. 70 Gy: $70.3 \%$ vs. $65.9 \%$ vs. $65.3 \%$, respectively; $p=0.964)$. Furthermore, as shown in Table 4, patients with a PTV $>60 \mathrm{~cm}^{3}$ demonstrated lower 2-year DFS than those with lower PTV; however, the difference was not statistically significant ( $\leq 60$ vs. $>60 \mathrm{~cm}^{3}$ : $40.6 \%$ vs. $25.0 \%$, respectively; $p=0.060$ ). Patients with Eastern Cooperative Oncology Group (ECOG) 0-1 demonstrated better OS than those with ECOG 2-3 (0-1 vs. 2-3: 2-year OS, 76.5\% vs. $50.0 \%$, respectively; $p=0.002$ ). However, other factors such as PTV volume, tumor size, radiation dose, and tumor location were not statistically associated with OS.

As illustrated in Fig. 3, dominant failure pattern was distant failure (51.3\%). Local failure was observed in $45.9 \%$ of the patients, and regional failure was observed in $40.5 \%$ of the patients.

\section{Toxicity}

Four patients (6.25\%) experienced grade 3 or higher toxicity. One patient died because of RT pneumonitis 3 months after the end of RT; two patients experienced grade 3 dyspnea after 5 and 10
Table 4. Univariate analysis for DFS and OS

\begin{tabular}{|c|c|c|c|c|c|}
\hline \multirow[b]{2}{*}{ Variable } & \multirow{2}{*}{$\begin{array}{l}\text { Number of } \\
\text { patients }\end{array}$} & \multicolumn{4}{|c|}{ Univariate analysis } \\
\hline & & $\begin{array}{c}\text { 2-yr DFS } \\
(\%)\end{array}$ & $p$-value & $\begin{array}{c}2-y r \text { OS } \\
(\%)\end{array}$ & $p$-value \\
\hline Age (yr) & & & 0.591 & & 0.289 \\
\hline$<70$ & 25 & 28.0 & & 60.0 & \\
\hline$\geq 70$ & 39 & 35.9 & & 66.7 & \\
\hline Sex & & & 0.058 & & 0.131 \\
\hline Male & 59 & 28.8 & & 61.0 & \\
\hline Female & 5 & 80.0 & & 80.0 & \\
\hline $\begin{array}{l}\text { ECOG performance } \\
\text { status }\end{array}$ & & & 0.709 & & 0.002 \\
\hline $0-1$ & 34 & 32.4 & & 76.5 & \\
\hline $2-3$ & 30 & 33.3 & & 50.0 & \\
\hline Histologic type & & & 0.698 & & 0.161 \\
\hline $\mathrm{SqCC}$ & 42 & 33.3 & & 59.5 & \\
\hline Others & 22 & 31.8 & & 72.7 & \\
\hline Tumor location & & & 0.302 & & 0.429 \\
\hline Central & 38 & 34.2 & & 68.4 & \\
\hline Non-central & 26 & 30.8 & & 57.7 & \\
\hline Tumor size (cm) & & & 0.164 & & 0.177 \\
\hline$\leq 5$ & 51 & 35.3 & & 68.6 & \\
\hline$>5$ & 13 & 23.1 & & 46.2 & \\
\hline PTV volume $\left(\mathrm{cm}^{3}\right)$ & & & 0.060 & & 0.325 \\
\hline$\leq 60$ & 32 & 40.6 & & 68.8 & \\
\hline$>60$ & 32 & 25.0 & & 59.4 & \\
\hline Dose (Gy) & & & 0.220 & & 0.546 \\
\hline $50-60$ & 51 & 31.4 & & 62.7 & \\
\hline $65-70$ & 13 & 38.5 & & 69.2 & \\
\hline
\end{tabular}

DFS, disease-free survival; OS, overall survival; ECOG, Eastern Cooperative Oncology Group; SqCC, squamous cell carcinoma; PTV, planning target volume.

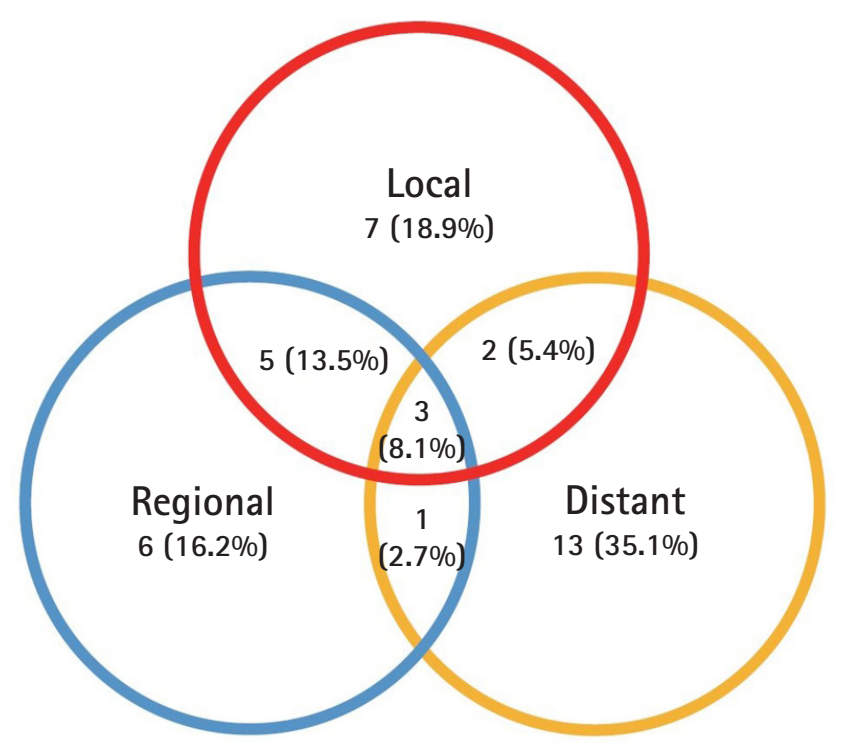

Fig. 3. Failure patterns of first recurrence. 
months, respectively; and one patient developed grade 3 RT pneumonitis 4 months after the treatment. All patients had pulmonary diseases, such as idiopathic pulmonary fibrosis (IPF) or chronic obstructive pulmonary disease (COPD). The only significant factor for grade 3 or higher toxicity was the presence of pulmonary disease, such as IPF or COPD ( $p=0.009)$. Other factors such as the sex, performance status, age, radiation dose, tumor location (central vs. non-central; ultracentral vs. non-ultracentral), and pre-treatment pulmonary functions test (forced expiratory volume in 1 second [FEV1], diffusing capacity of the lungs for carbon monoxide [DLCO]) did not demonstrate any significant difference in grade 3-5 toxicity. One patient who died of RT pneumonitis had underlying IPF. After HFRT of 60 Gy in 10 fractions, he received SBRT of 48 Gy in 4 fractions for contralateral lung mass. Three months after HFRT and about 1 month after SBRT, he was treated for pneumonia and RT pneumonitis. After discharge, he stopped taking antibiotics and steroids on his own. Despite rehospitalization and supportive care due to dyspnea aggravation, he died of respiratory arrest.

\section{Discussion and Conclusion}

This study was a single-institution retrospective analysis of patients treated with HFRT with primary or recurrent NSCLC to evaluate the local control rates, survival, and related toxicities. The 2-year FFLP, DFS, and OS rates were $67.8 \%, 32.8 \%$, and $64.1 \%$, respectively, and four patients (6.25\%) experienced grade 3 or higher toxicity. Given the acceptable toxicity, our findings provide clues regarding the optimal hypofractionation regimen for patients who are unfit for SBRT.

Historically, studies with conventional RT have reported local control rates of 30\%-70\% [14-17]. To improve the local control rate and OS, SBRT was attempted in early-stage lung cancer and demonstrated high local control (above 90\%) in multi-center, prospective trials [3-5]. However, since tumors that are central, large, and adjacent to ribs demonstrated high toxicity rates when SBRT was performed, risk-adapted fractionation schemes such as HFRT have been attempted to reduce the toxicity while trying to maintain the local control rate. However, a consensus regarding the schemes remains lacking. Table 5 summarizes the clinical outcomes of HFRT in various institutions [18-23]. Tekatli et al. $[18,19]$ reported that $60 \mathrm{~Gy}$ in 8 fractions in the treatment of central tumors, but not ultracentral tumors, had comparable OS with SBRT (2-year OS $=62 \%$ ); however, 60 Gy in 12 fractions in the treatment of ultracentral tumors resulted in 38\% grade 3-5 toxicity, although local failure was not observed during the follow-up. NRG Oncology/ RTOG 0813 tried a dose-escalating schedule in 5 fractions; 60 Gy in the 5-fraction schedule revealed $7.2 \%$ grade 3-5 toxicity with $87.9 \%$ of 2-year local control rates [20]. In contrast, the Nordic HILUS trial, a prospective multi-center phase II trial, reported as an abstract, tried 56 Gy in 8 fractions for central tumors and reported $28 \%$ grade $3-5$ toxicities with $9.5 \%$ grade 5 toxicity and more frequently toxicities in tumors close to the main bronchus than those close to a lobar bronchus [21]. RTOG 0813 included a relatively small proportion of ultracentral tumors (17\%) compared with our study (45.6\%) and defined a central tumor as a lesion within $2 \mathrm{~cm}$ from the proximal bronchial tree (PBT), while the HILUS trial defined it as a tumor within $1 \mathrm{~cm}$ from PBT; therefore, that could have resulted in the low toxicity rates in RTOG 0813 trial. Additionally, Li et al. [22] demonstrated 96.2\% 2-year LC with 3.6\% toxicity using $70 \mathrm{~Gy}$ in 10 fractions. In this study, approximately half the patients had central tumors and included patients with tumors ad-

Table 5. Clinical outcomes of hypofractionated radiation therapy in non-small cell lung cancer

\begin{tabular}{|c|c|c|c|c|c|c|}
\hline Study, year & $n$ & Dose (BED) & Median follow-up (mo) & Local control & OS & Toxicity $\geq$ Gr3 \\
\hline \multirow[t]{2}{*}{ Bezjak et al. [20], 2019} & 120 & $50-60 \mathrm{~Gy} / 5 \mathrm{fx}$ & 37.1 & 1-yr: 97\% & 1-yr: 93.9\% & DLT: $7.2 \%$ \\
\hline & & (100-132 Gy) & & 2-yr: 87.9\% & 2-yr: $72.7 \%$ & \\
\hline Lindberg et al. [21], 2017 & 74 & $\begin{array}{c}56 \text { Gy/8 fx } \\
\text { (95.2 Gy) }\end{array}$ & NA & NA & NA & $28 \%$ \\
\hline \multirow[t]{2}{*}{ Tekatli et al. [19], 2015} & 80 & $60 \mathrm{~Gy} / 8 \mathrm{fx}$ & 47 & NA & $1-y r: 81 \%$ & 13.9\% (Gr5: 7.5\%) \\
\hline & & (105 Gy) & & & $2-y r: 62 \%$ & \\
\hline \multirow[t]{2}{*}{ Tekatli et al. [18], 2016} & 47 & $60 \mathrm{~Gy} / 12 \mathrm{fx}$ & 29.3 & No LR & $1-y r: 61.5 \%$ & $38 \%$ \\
\hline & & (90 Gy) & & & $2-y r: 28.7 \%$ & \\
\hline Stephans et al. [23], 2017 & 33 & $\begin{array}{c}60 \text { Gy/8 fx } \\
\text { (105 Gy) }\end{array}$ & 22.1 & $2-y r: 87 \%$ & $2-y r: 52 \%$ & $15.1 \%$ \\
\hline Li et al. [22], 2014 & 82 & $\begin{array}{c}70 \text { Gy/10 fx } \\
\text { (119 Gy) }\end{array}$ & 21.1 & $2-y r: 96.2 \%$ & $2-y r: 66.9 \%$ & $3.6 \%$ \\
\hline \multirow[t]{2}{*}{ Current study } & 68 & $50-70 \mathrm{~Gy} / 10 \mathrm{fx}$ & 25.5 & 1-yr: 79.8\% & $1-y r: 82.8 \%$ & $6.3 \%$ \\
\hline & & (75-119 Gy) & & 2-yr: 67.8\% & $2-y r: 64.1 \%$ & \\
\hline
\end{tabular}

BED, biologic equivalent dose; OS, overall survival; Gr, grade; DLT, dose-limiting toxicity; NA, not available. 
jacent to the chest wall, large tumors, or multiple lesions. More than half the tumors were $<3 \mathrm{~cm}$, which could have resulted in a high control rate with comparative low toxicity.

In this study, univariate analysis demonstrated that escalation of the total dose did not have a significant association with local control, DFS, and OS. It has been demonstrated in several studies that SBRT regimens with $B E D \geq 100$ Gy have better local control and survival rates than with BED < $100 \mathrm{~Gy}$; if BED $\geq 100$ Gy was used, the local control rates were $>85 \%$ [24-26]. In this study, at the beginning of the treatment of the 10-fraction HFRT regimen due to central location or normal tissue constraints, we were reluctant to use a BED of > 100 Gy because of a lack of experience. However, following increase in experience and several reports regarding the safety of HFRT, dose escalation to 65-70 Gy has been attempted. However, since the sample size was not large, high-dose HFRT was performed relatively recently and treated patients had heterogeneous characteristics; therefore, these factors might have resulted in no significant difference in the local control with escalating total dose. Further studies with longer follow-up and large sample size are needed. Also, considering the low rates of toxicity in 10-fraction HFRT, there is room for improving local control by escalating the total dose or fraction size. In contrast, this study demonstrated no difference in toxicity above grade 3 between ultracentral and non-ultracentral lesions; however, this may be due to factors such as low toxicity rate and small sample size. Since it has been demonstrated in several studies that SBRT or HFRT for ultracentral lesions is likely to be highly toxic, it is necessary to carefully determine the fraction size and total dose, especially, for ultracentral lesions. Furthermore, in univariate analysis, the PTV volume, but not the tumor size, was statistically associated with local progression; however, it was not associated with DFS and OS. Similarly, Allibhai et al. [9] reported that the tumor size was not associated with local progression but was associated with DFS and OS and that the effects of tumor volume on DFS and OS were more significant than those of tumor size.

Besides, considering low DFS and OS after HFRT and that the most common failure pattern was distant metastasis, adjuvant treatment such as chemotherapy or immunotherapy could be helpful in disease control. The PACIFIC trial demonstrated that treatment with the immune checkpoint inhibitor durvalumab after CCRT demonstrated benefits in OS and DFS in patients with stage III unresectable NSCLC $[27,28]$. Therefore, immunotherapy or chemotherapy after HFRT could be beneficial in DFS and OS, and further studies regarding the adjuvant treatments are warranted.

Our study has a few limitations. It was a retrospective study at a single institution, which could have resulted in potential selection bias. Additionally, the heterogeneity of patients, tumor locations, and various organs at risk could have affected the planning and delivery of RT and, consequently, the clinical outcomes. Despite these limitations, this study included a relatively long follow-up compared with other studies on HFRT. Additionally, as there are few studies on the clinical outcomes of 10-fraction HFRT, this study could help predict the prognosis of the patients treated with this HFRT regimen.

In conclusion, HFRT with 50-70 Gy in 10 fractions demonstrated acceptable toxicity, although the local control rate appears to have a room for improvement via escalation of the total dose or fraction size and, especially, ultracentral tumors require more attention due to concerns of toxicity. For patients who are unfit for SBRT, more studies are required to investigate the optimal fractionation scheme.

\section{Conflict of Interest}

No potential conflict of interest relevant to this article was reported.

\section{References}

1. Videtic GM, Donington J, Giuliani M, et al. Stereotactic body radiation therapy for early-stage non-small cell lung cancer: executive summary of an ASTRO ASTRO Evidence-Based Guideline. Pract Radiat Oncol 2017;7:295-301.

2. Guckenberger $M$, Andratschke N, Dieckmann $K_{1}$ et al. ESTRO ACROP consensus guideline on implementation and practice of stereotactic body radiotherapy for peripherally located early stage non-small cell lung cancer. Radiother Oncol 2017;124:117.

3. Timmerman R, Paulus R, Galvin J, et al. Stereotactic body radiation therapy for inoperable early stage lung cancer. JAMA 2010; 303:1070-6.

4. Bezjak A, Paulus R, Gaspar LE, et al. Efficacy and toxicity analysis of NRG Oncology/RTOG 0813 trial of stereotactic body radiation therapy (SBRT) for centrally located non-small cell lung cancer (NSCLC). Int J Radiat Oncol Biol Phys 2016;96:S8.

5. Videtic GM, Paulus $R$, Singh $A K$, et al. Long-term follow-up on NRG Oncology/RTOG 0915 (NCCTG N0927): a randomized phase 2 study comparing 2 stereotactic body radiation therapy schedules for medically inoperable patients with stage I peripheral non-small cell lung cancer. Int J Radiat Oncol Biol Phys 2019; 103:1077-84.

6. Timmerman R, McGarry R, Yiannoutsos C, et al. Excessive toxicity when treating central tumors in a phase II study of stereotactic body radiation therapy for medically inoperable early-stage lung cancer. J Clin Oncol 2006;24:4833-9. 
7. Song SY, Choi W, Shin SS, et al. Fractionated stereotactic body radiation therapy for medically inoperable stage I lung cancer adjacent to central large bronchus. Lung Cancer 2009;66:89-93.

8. Kim SS, Song SY, Kwak J, et al. Clinical prognostic factors and grading system for rib fracture following stereotactic body radiation therapy (SBRT) in patients with peripheral lung tumors. Lung Cancer 2013;79:161-6.

9. Allibhai Z, Taremi M, Bezjak A, et al. The impact of tumor size on outcomes after stereotactic body radiation therapy for medically inoperable early-stage non-small cell lung cancer. Int J Radiat Oncol Biol Phys 2013;87:1064-70.

10. Ong CL, Palma D, Verbakel WF, Slotman BJ, Senan S. Treatment of large stage I-II lung tumors using stereotactic body radiotherapy (SBRT): planning considerations and early toxicity. Radiother Oncol 2010;97:431-6.

11. McGarry RC, Papiez L, Williams M, Whitford T, Timmerman RD. Stereotactic body radiation therapy of early-stage non-small-cell lung carcinoma: phase I study. Int J Radiat Oncol Biol Phys 2005;63:1010-5.

12. Baumann $P$, Nyman J, Hoyer $M$, et al. Outcome in a prospective phase II trial of medically inoperable stage I non-small-cell lung cancer patients treated with stereotactic body radiotherapy. J Clin Oncol 2009;27:3290-6.

13. Chi A, Liao Z, Nguyen NP, Xu J, Stea B, Komaki R. Systemic review of the patterns of failure following stereotactic body radiation therapy in early-stage non-small-cell lung cancer: clinical implications. Radiother Oncol 2010;94:1-11.

14. Coy P, Kennelly GM. The role of curative radiotherapy in the treatment of lung cancer. Cancer 1980;45:698-702.

15. Armstrong JG, Minsky BD. Radiation therapy for medically inoperable stage I and II non-small cell lung cancer. Cancer Treat Rev 1989;16:247-55.

16. Haffty BG, Goldberg NB, Gerstley J, Fischer DB, Peschel RE. Results of radical radiation therapy in clinical stage I, technically operable non-small cell lung cancer. Int J Radiat Oncol Biol Phys 1988;15:69-73.

17. Qiao X, Tullgren O, Lax I, Sirzen F, Lewensohn R. The role of radiotherapy in treatment of stage I non-small cell lung cancer. Lung Cancer 2003;41:1-11.

18. Tekatli H, Haasbeek N, Dahele M, et al. Outcomes of hypofrac- tionated high-dose radiotherapy in poor-risk patients with "ultracentral" non-small cell lung cancer. J Thorac Oncol 2016;11: 1081-9.

19. Tekatli H, Senan S, Dahele M, Slotman BJ, Verbakel WF. Stereotactic ablative radiotherapy (SABR) for central lung tumors: plan quality and long-term clinical outcomes. Radiother Oncol 2015; 117:64-70.

20. Bezjak A, Paulus R, Gaspar LE, et al. Safety and efficacy of a five-fraction stereotactic body radiotherapy schedule for centrally located non-small-cell lung cancer: NRG Oncology/RTOG 0813 Trial. J Clin Oncol 2019;37:1316-25.

21. Lindberg K, Bergstrom P, Brustugun OT, et al. OA24.05 The Nordic HILUS-Trial - first report of a phase ii trial of SBRT of centrally located lung tumors. J Thorac Oncol 2017;12(suppl):S340.

22. Li Q, Swanick CW, Allen PK, et al. Stereotactic ablative radiotherapy (SABR) using 70 Gy in 10 fractions for non-small cell lung cancer: exploration of clinical indications. Radiother Oncol 2014; $112: 256-61$.

23. Stephans KL, Woody NM, Reddy CA, et al. Tumor control and toxicity for common stereotactic body radiation therapy dose-fractionation regimens in stage I non-small cell lung cancer. Int J Radiat Oncol Biol Phys 2018;100:462-9.

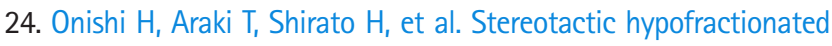
high-dose irradiation for stage I nonsmall cell lung carcinoma: clinical outcomes in 245 subjects in a Japanese multiinstitutional study. Cancer 2004;101:1623-31.

25. Mehta N, King CR, Agazaryan N, Steinberg M, Hua A, Lee P. Stereotactic body radiation therapy and 3-dimensional conformal radiotherapy for stage I non-small cell lung cancer: a pooled analysis of biological equivalent dose and local control. Pract Radiat Oncol 2012;2:288-95.

26. Senthi S, Haasbeek CJ, Slotman BJ, Senan S. Outcomes of stereotactic ablative radiotherapy for central lung tumours: a systematic review. Radiother Oncol 2013;106:276-82.

27. Antonia SJ, Villegas A, Daniel D, et al. Overall survival with durvalumab after chemoradiotherapy in stage III NSCLC. N Engl J Med 2018;379:2342-50.

28. Antonia SJ, Villegas A, Daniel D, et al. Durvalumab after chemoradiotherapy in stage III non-small-cell lung cancer. N Engl J Med 2017;377:1919-29. 